\title{
COVID-19 vaccine hesitancy in patients with systemic autoimmune rheumatic disease: an interview-based survey
}

\author{
Priyanka Gaur $^{1} \cdot$ Hardik Agrawat $^{1} \cdot$ Anuj Shukla ${ }^{1}{ }^{\mathbb{C}}$
}

Received: 2 May 2021 / Accepted: 28 June 2021 / Published online: 2 July 2021

(c) The Author(s), under exclusive licence to Springer-Verlag GmbH Germany, part of Springer Nature 2021

\begin{abstract}
Patients with a systemic autoimmune rheumatic disease (AIRD) are vulnerable to SARS Cov-2 infection. Vaccination against this infection can prevent the patients from developing severe disease. But vaccine hesitancy in this group can emerge as a hurdle. So there is a need to understand the perception regarding vaccination in AIRD patients. The study is an interviewbased survey done in AIRD patients and a control group from the general population. The questionnaire included the subject's demographic details, duration, diagnosis, the activity of AIRD, and questions regarding the perception of the vaccination. The survey included 280 patients with AIRD and 102 control subjects. 54\% (152/280) of the patients and 67\% (68/102) of the controls were willing to get vaccinated $(p=0.03)$. Patients $>45$-years of age were more willing to vaccinate than those with age $\leq 45$-years $(61.9 \%$ vs. $44.8 \% ; p=0.001)$. Patients with lower education had more vaccine hesitancy than those with graduation and above ( $38 \%$ vs. $69 \% ; p<0.001)$. The common reason for vaccine hesitancy was not-yet-decided, fear related to vaccine side-effects, and disease worsening. 29\% (82/280) patients were already vaccinated, out of which 35\% (35/82) had mild events (fever/myalgia/headache). AIRD patients had fewer side effects than controls, and disease flare was seen in only one patient. Thus, educating AIRD patients regarding the pros and cons of vaccination, particularly concerning immunological disease, can help us overcome vaccine hesitancy. The message should clearly penetrate that there is a negligible risk of AIRD-flares with the COVID-19 immunization and the side effects are mild and manageable.
\end{abstract}

Keywords COVID-19 vaccination · Rheumatic disease $\cdot$ Vaccine hesitancy

\section{Introduction}

The coronavirus disease 2019 (COVID-19) has proven to be the biggest threat to individuals worldwide. India is facing a huge second wave of illness. Apart from COVID-19 appropriate behaviour, mass vaccination is the only measure to control the ferocity of the pandemic. The rapid development of the vaccine, an emergency approval by the government, and many other socio-cultural factors in India resulted in vaccine hesitancy. World Health Organisation (WHO) Strategic Advisory Group of Experts (SAGE) on immunization has described vaccine hesitancy as 'delay in acceptance or refusal of vaccination despite the availability of vaccination services' [1]. It depends upon multiple factors, significantly on confidence (trust on vaccine), complacency (do

Anuj Shukla

anuj.guj@gmail.com

1 Present Address: Niruj Rheumatology Clinic, 209 Rajvi Complex, Rambaug, Ahmedabad 380008, Gujarat, India not perceive the need of vaccine), and convenience (ease of accessibility) [2]. WHO has listed vaccine hesitancy as one of the top ten threats to global health in 2019.

Patients with systemic autoimmune rheumatic disease (AIRD) are a vulnerable group for SARS Cov-2 infection [3-5]. The heterogeneous nature of AIRD and treatment with various immunomodulators can lead to an immunocompromised state. Thus, increasing the vulnerability to the virus infection and can even lead to severe disease. Their perception towards the vaccination and immunological response can also vary compared to the general population. During the second phase of the mass vaccination program, the Government of India advised vaccination to all citizens above 60 years of age. The AIRD patients aged above 45 years were considered a vulnerable group and recommended vaccination.

A global survey conducted on the general population and health workers in June 2020 has reported the vaccine acceptance rate varying from $90 \%$ in China to less than $55 \%$ in Russia and $74.5 \%$ in India [6]. There are many myths among 
the Indian population regarding COVID vaccination. The common ones are the vaccine can lead to death, those vaccinated will survive for a maximum of two years, and the vaccine can even lead to impotence. This hesitancy seems to be a significant coming hurdle while vaccinating the entire population. These perceptions in the people, particularly in the AIRD-patients as a vulnerable group, need to be looked at carefully to overcome vaccine hesitancy in the future.

\section{Methods}

The study is an interview-based survey. It was done from 22nd March to 22nd April 2021 at a rheumatology center in western India. An ACEAS-independent Ethics Committee, Gujarat, approved the study protocol number 02/2021/05 on 21st May 2021. The study included consecutive patients with the diagnosis of AIRD visiting the clinic. Patients with less than 18-years of age, degenerative joint diseases, isolated radiculopathies, and other soft-tissue rheumatism unrelated to AIRD were excluded from the study. The control population was selected from the neighbours of five staff members. The interviewer visited the houses in the evening hours and included all the members greater than 18-years of age as control. The relatives of the patients were not selected as a control group to prevent any perceived bias towards the vaccination. The interviewer visited 25 houses, persons ( $>18$ years of age) were included as a control in the study. None of the control was a known case of AIRD and was not on any immunomodulator medicines. Written informed consent was taken from all the patients and the controls.

A single interviewer did a face-to-face structured interview for all the patients and control using a prepared questionnaire (supplementary material). The questionnaire was in the English language. The interviewer (author-2) initially did a short survey of 20-patients. The authors collectively then made the necessary changes in the language of the questionnaire to ensure the validity of the questions before finalizing the document. However, this exercise did not include an external expert panel or an extensive process of validation [7]. An effort was made to form the questions in a simplified manner, with the answers limited to yes/no (close-ended questions) except for the question looking into the cause for vaccine hesitancy. The questionnaire included the subject's particulars, education, comorbidities, and the questions regarding the knowledge, perception, and attitude of the subjects regarding the vaccination. The document also included the diagnosis of AIRD, duration of disease and follow-up, the disease activity status. The data was collected and analyzed using the SPSS Statistics software for windows, Version-16 Chicago: SPSS Inc. Statistical analyses were done using $\chi^{2}$-test and binary logistic regression analyses ( $p$-value: two-sided, $<0.05$ ).

\section{Results}

Three hundred eighty-two subjects participated in the survey; 280 were AIRD patients, and 102 were controls. Table 1 shows the demographic details of both groups. In total, $57.5 \%$ (220/382) of the subjects were ready to get the vaccine, the willingness was 54\% (152/280) among the patients and $67 \%$ $(68 / 102)$ among the controls $\left.\left[\chi^{2}(1, \mathrm{~N}=382)=4.7, p=0.035\right]\right)$.

\section{Predictors and reasons of vaccination hesitancy in patients}

Patients $>45$-years of age were more willing than those with age less than or equal to 45 -years [ $61.9 \%$ vs. $44.8 \%$; OR 2.8 (95\% CI 1.5-5.2) $p=0.001]$. In comparison to patients with lower education (incomplete school or no education), patients with complete school education (53\% vs. 38\%; OR 2.1 (95\% CI 1.07-4.16) $p=0.031$ ) and those with graduation and above (69\% vs. $38 \%$; OR 4.6 (95\% CI $2.13-10.12) p<0.001$ ). There was no difference among the patients with various AIRD diagnosis subgroups. Also, understanding of the COVID-19 or AIRD disease by patients, disease activity status, the duration of the disease, or the treatment had no significant effect on the vaccine hesitancy.46\% (128/280) patients and 33\% (34/102) controls had shown vaccine hesitancy. Table 2 offers the common reasons for such reluctance.

\section{Adverse events in vaccinated patients and controls}

The total number of patients and controls who have already received the vaccine was 29\% (82/280) and 52\% (53/102), respectively. All the vaccinated subjects received ChAdOx1 nCoV-19 Corona Virus Vaccine (Recombinant) Covishield ${ }^{\mathrm{TM}}$. Adverse events were seen in 35\% (29/82) of the patients and $68 \%(36 / 53)$ of the controls $(p=0.0002)$. Figure 1 shows the frequency of various adverse events in both groups. A patient with seropositive rheumatoid arthritis had a post-vaccination flare (within 14 days) requiring a short course of low-doseprednisolone. Most of these patients (78/82) have taken the vaccine themselves and did not consider the necessity of a prior discussion with the physician or the treating rheumatologist. There was a marked lack of awareness regarding pneumococcal or influenza vaccination (2/280 - aware of these vaccines) in the AIRD patients.

\section{Discussion}

The total willingness to take the COVID vaccine was 57.5\% and was lesser among the AIRD patients than the controls. Similar observations were seen in another study from Rome with a low acceptance in AIRD-patients (54.9\%) compared 
Table 1 Demographic details of patients diagnosed with systemic autoimmune rheumatic diseases and controls

\begin{tabular}{|c|c|c|c|}
\hline & AIRD patients $n(\%)$ & Controls $n(\%)$ & $p$-value \\
\hline Number & 280 & 102 & \\
\hline Mean \pm SD Age (years) & $47 \pm 13$ & $49 \pm 12$ & 0.17 \\
\hline Co-morbidity (hypertension/DM) & $68(24)$ & $17(17)$ & 0.11 \\
\hline $\mathrm{F}: \mathrm{M}$ & $5: 1$ & $1: 1$ & $<0.0001 *$ \\
\hline Education & $n=229 *$ & & \\
\hline$<$ Primary & $104(45)$ & $16(16)$ & $<0.0001^{*}$ \\
\hline Higher and Senior secondary & $71(31)$ & $33(32)$ & 0.80 \\
\hline$>$ Graduate & $54(24)$ & $53(52)$ & $<0.0001^{*}$ \\
\hline \multicolumn{4}{|l|}{ Disease group } \\
\hline Rheumatoid Arthritis (RA) & $200(71)$ & - & \\
\hline Seropositive $R A$ & 133 & & \\
\hline Seronegative $R A$ & 57 & & \\
\hline Undifferentiated arthritis & 10 & & \\
\hline Spondyloarthritis & $27(10)$ & - & \\
\hline Other Systemic autoimmune disease & $53(19)$ & - & \\
\hline Systemic Lupus Erythematosus & 14 & & \\
\hline Idiopathic Inflammatory Myositis & 8 & & \\
\hline Mixed connective tissue disease & 8 & & \\
\hline Fibromyalgia & 8 & & \\
\hline Systemic Sclerosis & 8 & & \\
\hline Vasculitis & 3 & & \\
\hline Sjogren's syndrome & 3 & & \\
\hline Gout & 3 & & \\
\hline Sarcoidosis & 1 & & \\
\hline \multicolumn{4}{|l|}{ Disease status } \\
\hline Active & $153(55)$ & - & \\
\hline Remission & $127(45)$ & - & \\
\hline \multicolumn{4}{|l|}{ Disease duration at the time of the study (years) } \\
\hline$<1$ year & $104(37)$ & - & \\
\hline $1-5$ years & $113(40)$ & - & \\
\hline$>5$ year & $63(23)$ & - & \\
\hline \multicolumn{4}{|l|}{ Treatment duration (years) } \\
\hline$<1$ year & $197(70)$ & - & \\
\hline $1-5$ years & $71(25)$ & - & \\
\hline$>5$ year & $13(5)$ & - & \\
\hline Knowledge about AIRD & $229(82)$ & - & \\
\hline Knowledge about COVID-19 & $278(99)$ & 102 & \\
\hline $\begin{array}{l}\text { Knowledge about Influenzae/Pneumococcal } \\
\text { vaccine }\end{array}$ & $2(0.7)$ & - & \\
\hline
\end{tabular}

AIRD autoimmune rheumatic disease, $D M$ diabetes mellitus

*Education was available for $\mathrm{n}-229$ patients with AIRD to controls $(82.3 \%)$ [8]. While studies from Turkey and Amsterdam showed no difference between the patients and control groups for vaccination willingness $[9,10]$. In our study, the higher willingness of the control group may be attributed to higher education in the group than the patient group. Another reason may be that patients suffering from immunological diseases were more skeptical regarding the effect of the vaccine on the immune system and their illness in particular. So, they were less willing to get vaccinated than control group subjects who do not have an immunological disease.

The patients above 45 -years of age and having higher education were more willing to take the vaccine. These observations were similar to the previous survey reports [8-12]. Additionally, few studies observed that males were more willing to get vaccinated than females $[9,10,12]$. However, 
Table 2 Reasons for vaccine hesitancy in patients and controls group

\begin{tabular}{lll}
\hline Reasons for vaccine hesitancy & Patients $n, \%$ & $\begin{array}{l}\text { Controls } n, \\
\% \text { Controls } \\
n, \%\end{array}$ \\
\hline Not-yet-decided & 49,38 & 28,82 \\
No need of vaccination & 34,26 & - \\
Fear related to vaccine side effects & 22,17 & 6,18 \\
Fear related to disease worsening & 15,12 & - \\
Distrust on vaccine & 4,3 & - \\
May not be effective & 2,2 & - \\
Others & 2,2 & - \\
\hline
\end{tabular}
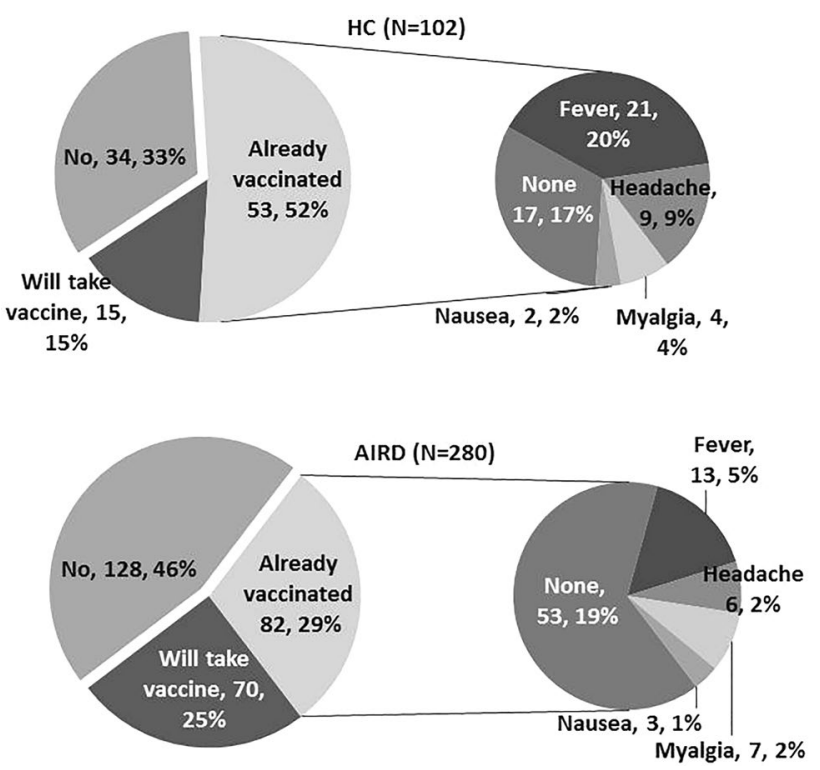

Fig. 1 Frequency of various adverse events in patients with systemic autoimmune rheumatic disease and the control groups

in our study, we have not observed any such difference. The positive attitude for vaccination in older individuals may be attributed to the awareness of more severe COVID-disease in older adults than younger individuals (complacency $=$ do not perceive the need for the vaccine). The phased vaccination program in India, allowing early vaccination among this age group, might have also contributed to their acceptance (convenience $=$ ease of availability). The effect of education on the willingness/hesitancy to take the vaccine may be related to the ease of ability to differentiate between the myths and facts of getting vaccinated (confidence $=$ trust on vaccine). There is extensive and rapidly evolving information available on various social and news platforms, which might be confusing, particularly for uneducated individuals.

In the present study, 38\% (49/128) of the patients were not-yet-decided about the immunization and thus constitute the main reason for vaccine refusal. Felten et al. and
Yurttas et al. had also reported the uncertainty for vaccination in their patient cohort, $32.2 \%$, and $51.8 \%$, respectively $[10,12]$. These studies also reported that willingness to get vaccinated could be increased if recommended by a specialist physician $[9,10,12]$. The other reasons for refusal were fear of adverse events, a flare-up of underlying AIRD, and doubt related to the vaccine efficacy, similar to those reported in previous studies $[8,9,11]$. These points show that the AIRD patients still lack clarity about the pros and cons of the vaccination. Thus, providing them accurate information by the doctors could help them to reconsider their decision.

The survey showed that vaccination in AIRD patients was safe with no significant events of disease flare. The adverse events of vaccination, such as fever, myalgia, were lesser in the studied population than in the controls. The frequent use of non-steroid anti-inflammatory drugs and steroids in the patients might have masked these side effects. The lack of awareness about influenza or pneumococcal vaccines highlights a marked deficiency in the knowledge and acceptance of adult immunization in the Indian population, particularly before the COVID-19 pandemic. The limitation of our study was the small sample size, lack of an extensive process of validation, and a self-reported questionnaire. However, the questionnaire was replicable and straightforward.

Thus, educating AIRD patients regarding the pros and cons of vaccination, particularly concerning immunological disease, can help us overcome vaccine hesitancy. The message should clearly penetrate that there is a negligible risk of AIRD-flares with the COVID-19 immunization and the side effects are mild and manageable.

Acknowledgements We would like to acknowledge all the participants of the study. Also we would like to thanks all the staff members of the clinic to help in recruiting controls for the study.

Author contributions PG, HA and AS has designed the study, made the proforma, collected and analysed the data. All authors have participated in writing and reviewing the manuscript and has provided all the necessary critical intellectual inputs. All authors take full responsibility for all aspects of the study. HA has taken the interview of all the subjects.

Funding Not applicable.

Availability of data and materials Data will be available to the journal if required.

Code availability Not applicable.

\section{Declarations}

Conflict of interest Authors declared no conflict of interest.

Ethics approval The study was approved by Independent Ethics Committee ACEAS-IEC, Ahmedabad, Gujarat. Protocol No. 02/2021/05, (Version No. 00, Dated 21-May-2021). 


\section{References}

1. MacDonald NE, SAGE Working Group on Vaccine Hesitancy (2015) Vaccine hesitancy: definition, scope, and determinants. Vaccine 33:4161-4164

2. Troiano G, Nardi A (2021) Vaccine hesitancy in the era of COVID-19. Public Health 194:245-251

3. Xu C, Yi Z, Cai R, Chen R, Thong BY, Mu R (2021) Clinical outcomes of COVID-19 in patients with rheumatic diseases: a systematic review and meta-analysis of global data. Autoimmun Rev 20:102778

4. Zhong J, Shen G, Yang H, Huang A, Chen X, Dong L (2020) COVID-19 in patients with rheumatic disease in Hubei province, China: a multicentre retrospective observational study. Lancet Rheumatol 2:e557-e564

5. Kipps S, Paul A, Vasireddy S (2021) Incidence of COVID-19 in patients with rheumatic disease: is prior health education more important than shielding advice during the pandemic? Clin Rheumatol 40:1575-1579

6. Lazarus JV, Ratzan SC, Palayew A et al (2021) A global survey of potential acceptance of a COVID-19 vaccine. Nat Med 27:225-228

7. Gaur PS, Zimba O, Agarwal V, Gupta L (2020) Reporting survey based studies-a primer for authors. J Korean Med Sci 35:398

8. Priori R, Pellegrino G, Colafrancesco S, Alessandri C, Ceccarelli F, Di Franco M et al (2021) SARS-CoV-2 vaccine hesitancy among patients with rheumatic and musculoskeletal diseases: a message for rheumatologists. Ann Rheum Dis 80:953-954

9. Boekel L, Hooijberg F, van Kempen ZLE, Vogelzang EH, Tas SW, Killestein J (2021) Perspective of patients with autoimmune diseases on COVID-19 vaccination. Lancet Rheumatol 3:e241-e243

10. Yurttas B, Poyraz BC, Sut N, Ozdede A, Oztas M, Uğurlu S, Tabak F, Hamuryudan V, Seyahi E (2021) Willingness to get the COVID-19 vaccine among patients with rheumatic diseases, healthcare workers and general population in Turkey: a web-based survey. Rheumatol Int 41:1105-1114

11. Campochiaro C, Trignani G, Tomelleri A, Cascinu S, Dagna L (2021) Potential acceptance of COVID-19 vaccine in rheumatological patients: a monocentric comparative survey. Ann Rheum Dis 3:e399-e401

12. Felten R, Dubois M, Ugarte-Gil MF et al (2021) Vaccination against COVID-19: expectations and concerns of patients with autoimmune and rheumatic diseases. Lancet Rheumatol 3:e243-e245

Publisher's Note Springer Nature remains neutral with regard to jurisdictional claims in published maps and institutional affiliations. 